\title{
First- and Second-Order Phase Transitions, Fulde-Ferrel Inhomogeneous State and Quantum Criticality in Ferromagnet/Superconductor Double Tunnel Junctions
}

\author{
Biao Jin, Gang Su* and Qing-Rong Zheng \\ College of Physical Sciences, Graduate School of the Chinese Academy of Sciences, P.O. Box 3908, Beijing 100039, China
}

\begin{abstract}
First- and second-order phase transitions, Fulde-Ferrel (FF) inhomogeneous superconducting (SC) state and quantum criticality in ferromagnet/superconductor/ferromagnet double tunnel junctions are investigated. For the antiparallel alignment of magnetizations, it is shown that a first-order phase transition from the homogeneous BCS state to the inhomogeneous FF state occurs at a certain bias voltage $V^{*}$; while the transitions from the BCS state and the FF state to the normal state at $V_{c}$ are of the second-order. A phase diagram for the central superconductor is presented. In addition, a quantum critical point (QCP), $V_{Q C P}$, is identified. It is uncovered that near the QCP, the SC gap, the chemical potential shift induced by the spin accumulation, and the difference of free energies between the SC and normal states vanish as $\left|V-V_{Q C P}\right|^{z \nu}$ with the quantum critical exponents $z \nu=1 / 2,1$ and 2, respectively. The tunnel conductance and magnetoresistance are also discussed.

PACS numbers: 73.40.Gk, 75.70.Pa, 73.40.Rw
\end{abstract}

Introduction. - Spin-dependent transport plays an essential role in magnetic hybrid nanostructures in the field of spintronics (see, e.g. Refs. [1, 2, 3, 4, 5, 6, 7] for review). Among others, ferromagnet/superconductor $(\mathrm{F} / \mathrm{S})$ heterostructures have attracted much attention theoretically 8, 9, 10, 11, 12, 13, 14 and experimentally 15, 16, 17, 18, 19, 20, 21] in recent years. For F/S/F double tunnel junctions, it has been observed that the superconductivity is suppressed by the injection of spin-polarized current (e.g. Refs. 9, 11, 12, 13, 14, 18, 20]), that is due to the nonequilibrium spin accumulation. When spin-polarized electrons are injected into the superconductor, a spin density is accumulated near the interfaces owing to the spin imbalance, thereby giving rise to an equivalent, small magnetic field that acts as a pair-breaking field, which leads to a suppression of superconductivity. There has been a recent study [9] showing that the homogeneous superconducting (SC) state is strongly suppressed with increasing the bias voltage and completely destroyed at a critical voltage by the nonequilibrium spin density in the antiparallel alignment of magnetizations. This study is qualitatively consistent with the experimental observation for high biases, but is inconsistent for low biases 18.

On the other hand, about forty years ago, Fulde and Ferrel (FF) 22], and Larkin and Ovchinnikov (LO) 23. independently, found that the SC order parameter can be modulated in real space by a spin-exchange field of a ferromagnet. Later, such an inhomogeneous superconducting state has been extensively explored under various circumstances (e.g. 17, 24]). As the nonequilibrium spin accumulation may lead to an equivalent magnetic field in the central supercondutor, the FFLO state, which is simply omitted in the previous treatment $[9]$, might be inevitable in the $\mathrm{F} / \mathrm{S} / \mathrm{F}$ double tunnel junction.

To understand profoundly the spin-dependent transport properties, in this Letter, the $\mathrm{F} / \mathrm{S} / \mathrm{F}$ double tunnel junction shall be systematically revisited. It is shown that in the antiparallel aligment, a first-order phase transition from a homogeneous Bardeen-Cooper-Schrieffer (BCS) SC state to the inhomogeneous FF SC state occurs at a certain bias voltage, while the transitions from the BCS state and the FF state to the normal state are of the second-order. A phase diagram for the central superconductor is identified. Besides, a quantum critical point (QCP) is specified at bias $V_{Q C P}$, near which the SC order parameter, the chemical potential shift induced by the spin accumulation, and the difference of free energies between the $\mathrm{SC}$ and normal states vanish as $\left|V-V_{Q C P}\right|^{z \nu}$ with the quantum critical exponents $z \nu=1 / 2,1$ and 2 , respectively. The tunnel conductance and magnetoresistance are also obtained.

Model. - Consider a symmetric F/S/F double tunnel junction with the left and right ferromagnetic (FM) electrodes applied by bias voltages $-V / 2$ and $V / 2$, respectively. The two identical FM electrodes are separated from the central superconductor by two insulating thin films. The central superconductor is presumably described within the framework of BCS theory. Suppose that the energy relaxation time of quasiparticles is shorter than the tunneling time, while the latter is shorter than the spin relaxation time. As the resistance of this tunnel junction with insulating thin films is greater than that of a conventional metallic contact, the Andreev reflection effect can be reasonably ignored for simplicity.

From the standard tunneling Hamiltonian, and in light of the linear response theory, the tunneling current through the $j$ th junction can be readily obtained by

$$
I_{j \sigma}=2 \pi e|\widetilde{T}|^{2} D_{j \sigma}\left[N-\eta_{j}\left(\sigma S+\frac{Q-\widetilde{N}}{2}\right)\right],
$$

where $\widetilde{T}$ is the tunneling matrix element, $j=1,2, D_{j \sigma}$ is the subband density of states (DOS) in the $j$ th FM electrode, $\sigma= \pm 1$ for spin up and down, respectively, and $\eta_{1}=1, \eta_{2}=-1$. The quantities $S, Q, N$ and $\widetilde{N}$ are defined by 


$$
\begin{aligned}
S & =\frac{1}{2} \sum_{\mathbf{k}}\left(f_{\mathbf{k} \uparrow}-f_{\mathbf{k} \downarrow}\right), \\
Q & =\frac{1}{2} \sum_{\mathbf{k}}\left(u_{\mathbf{k}}^{2}-v_{\mathbf{k}}^{2}\right)\left(f_{\mathbf{k} \uparrow}+f_{\mathbf{k} \downarrow}\right), \\
N & =\frac{1}{2} \sum_{\mathbf{k}}\left[f_{0}\left(E_{\mathbf{k}}-\frac{e V}{2}\right)-f_{0}\left(E_{\mathbf{k}}+\frac{e V}{2}\right)\right] \\
\widetilde{N} & =\frac{1}{2} \sum_{\mathbf{k}}\left(u_{\mathbf{k}}^{2}-v_{\mathbf{k}}^{2}\right)\left[f_{0}\left(E_{\mathbf{k}}-\frac{e V}{2}\right)+f_{0}\left(E_{\mathbf{k}}+\frac{e V}{2}\right)\right](5)
\end{aligned}
$$

where $f_{0}(z)$ denotes the Fermi distribution function of thermal equilibrium in FM electrodes, $f_{\mathbf{k} \sigma}$ is the nonequilibrium distribution function of quasiparticles with energy $E_{\mathbf{k}}$ and spin $\sigma(=\uparrow, \downarrow)$ in the central superconductor, $S$ and $Q$ represent the spin density and the quasiparticle charge density, describing the spin imbalance and quasiparticle charge imbalance in the central superconductor, respectively.

Fulde-Ferrel State. - As there appears the nonequilibrium spin accumulation near the interfaces of the tunnel junction, it is believed that the SC state would generally include the inhomogeneous FFLO state 22, 23] in addition to the homogeneous BCS state. Without loss of generality, we suppose that the SC order parameter takes the form of FF type [22]: $\Delta(\mathbf{r})=\Delta_{\mathbf{q}} e^{i \mathbf{q} \cdot \mathbf{r}}$ with $\Delta_{\mathbf{q}}$ the amplitude of the order parameter. When $\mathbf{q}=0$, it recovers the homogeneous BCS state. The quasiparticle dispersion $E_{\mathbf{k}}$, the coherence factors $u_{\mathbf{k}}$ and $v_{\mathbf{k}}$ are given by $E_{\mathbf{k}}=\sqrt{\xi_{\mathbf{k}}^{2}+\Delta_{\mathbf{q}}^{2}}+\left(v_{F} q / 2\right) x, u_{\mathbf{k}}^{2}=\frac{1}{2}\left(1+\xi_{\mathbf{k}} / \sqrt{\xi_{\mathbf{k}}^{2}+\Delta_{\mathbf{q}}^{2}}\right)$, and $v_{\mathbf{k}}^{2}=\frac{1}{2}\left(1-\xi_{\mathbf{k}} / \sqrt{\xi_{\mathbf{k}}^{2}+\Delta_{\mathbf{q}}^{2}}\right)$, respectively, where $\xi_{\mathbf{k}}$ is the free electron energy relative to the chemical potential, $v_{F}$ the Fermi velocity and $x=\mathbf{k} \cdot \mathbf{q} /(q k)$ the cosine of the angle between $\mathbf{k}$ and momentum $\mathbf{q}$ of a Cooper pair. The amplitude $\Delta_{\mathbf{q}}$ is determined by the gap equation

$$
1=\frac{V_{B C S}}{2} \sum_{\mathbf{k}} \frac{\left(1-f_{\mathbf{k} \uparrow}-f_{\mathbf{k} \downarrow}\right)}{\sqrt{\xi_{\mathbf{k}}^{2}+\Delta_{\mathbf{q}}^{2}}},
$$

where $V_{B C S}$ is the BCS-type pair interaction. The values of $\mathbf{q}$ will be specified later.

Let us proceed to determine the nonequilibrium distribution function $f_{\mathbf{k} \sigma}^{F(A)}$. In the absence of spin-flip scattering, the spin up and down tunneling currents are independent, and should be conserved, i.e. $I_{1 \sigma}=I_{2 \sigma}$, yielding

$$
\begin{aligned}
& S^{F}=0 \text { for the parallel alignment, } \\
& S^{A}=P N^{A} \text { for the antiparallel alignment, } \\
& Q^{F}=Q^{A}=0 \text { for both alignments, }
\end{aligned}
$$

where the superscripts $F$ and $A$ refer to the parallel and antiparallel alignments, respectively, and $P=$ $\left|D_{j \uparrow}-D_{j \downarrow}\right| /\left(D_{j \uparrow}+D_{j \downarrow}\right)$ is the spin polarization of the
FM electrodes. These solutions show that the nonequilibrium spin accumulation exists only in the antiparallel configuration. In the above derivation, we have adopted the conventional constant DOS approximation, $\sum_{\mathbf{k}}(\cdots) \simeq N(0) \int_{-\varpi_{D}}^{\varpi_{D}}(\cdots) d \xi_{\mathbf{k}}$, where $\varpi_{D}$ is the cut-off (Debye) energy, and $N(0)$ denotes the DOS of free electrons at the Fermi level. The quantity $\widetilde{N}$ vanishes identically for both alignments of magnetizations since the integrand is an odd function of $\xi_{\mathbf{k}}$. Eq. (8) requires that $f_{\mathbf{k} \uparrow}^{A}$ should differ from $f_{\mathbf{k} \downarrow}^{A}$ in the presence of the tunneling current. Following Ref. [9], we consider the solutions of the form:

$$
\begin{aligned}
& f_{\mathbf{k} \sigma}^{A}=f_{0}\left(E_{\mathbf{k}}-\sigma \delta \mu\right), \\
& f_{\mathbf{k} \uparrow}^{F}=f_{\mathbf{k} \downarrow}^{F}=f_{0}\left(E_{\mathbf{k}}\right),
\end{aligned}
$$

where $\delta \mu$ is introduced as the chemical potential shift induced by the nonequilibrium spin accumulation, and plays essentially the same role as the spin-exchange field explored by FF in their seminal article 22]. This kind of solutions may be applicable if the thickness of the central superconductor is much smaller than the spin diffusion length, and the spin relaxation time is sufficiently long. Eqs. (10) and (11) are the solutions of Eqs. (7) and (9). However, Eqs. (8) and (6) with Eqs. (10) should be solved in a self-consistent manner to specify $\delta \mu$ and $\Delta_{\mathbf{q}}$ as functions of the bias $V$, temperature $T$ and polarization $P$.

For these coupled equations, when the self-consistent multiple solutions corresponding to different $\mathbf{q}$ appear, only the value of $\mathbf{q}$ that leads to the lowest free energy of the system is retained. In an inhomogeneous superconducting state, the nonzero solution for $\Delta_{\mathbf{q}}$ implies only the local minimum of the free energy, which does not necessarily mean the stable state. In order to clarify this issue, one must compare the free energies of the homogeneous BCS, the inhomogeneous FF and normal states, as emphasized by Abrikosov 25. The free energy of the present system can be obtained by integrating the gap equation [26]

$$
\begin{aligned}
F_{S}^{F(A)}-F_{N}^{F(A)} & =\left(\Delta_{\mathbf{q}}^{F(A)}\right)^{2} / V_{B C S} \\
& -\int_{0}^{\Delta_{\mathbf{q}}^{F(A)}} d z \sum_{\mathbf{k}}\left(z / \sqrt{\xi_{\mathbf{k}}^{2}+z^{2}}\right) \\
& \times\left[1-f_{0}\left(\sqrt{\xi_{\mathbf{k}}^{2}+z^{2}}+\frac{v_{F}(\mathbf{q} \cdot \mathbf{k})}{2 k}-\delta \mu^{F(A)}\right)\right. \\
& \left.-f_{0}\left(\sqrt{\xi_{\mathbf{k}}^{2}+z^{2}}+\frac{v_{F}(\mathbf{q} \cdot \mathbf{k})}{2 k}+\delta \mu^{F(A)}\right)\right](12)
\end{aligned}
$$

where $F_{S}^{F(A)}$ and $F_{N}^{F(A)}$ stand for the free energy of the $\mathrm{SC}$ state and the $\mathrm{N}$ state, respectively, and $\delta \mu^{F}=0$, $\delta \mu^{A}=\delta \mu$. It turns out that both the homogeneous BCS $(\mathbf{q}=0)$ and the FF $(\mathbf{q} \neq 0)$ SC solutions are possible in the antiparallel alignment, while the former solution is always favorable in the parallel configuration. 
Results. - In the parallel alignment of magnetizations, since there is no spin and charge accumulation in this circumstance, the SC order parameter does not depend on the bias voltage; while in the antiparallel configuration, the situation becomes complicated, as the nonequilibrium spin accumulation characterized by $S^{A}$ intervenes in. Figure 1 presents the bias voltage dependence of the SC order parameter and the chemical potential shift at $T / T_{c}=0.2$, with $T_{c}$ the $\mathrm{SC}$ critical temperature, for the antiparallel alignment. It is observed that the order parameter $\Delta^{A}$ remains almost constant at low biases and is in the homogeneous BCS state till a specific bias voltage $V^{*}=1.36 \Delta_{0} / e$ at which $\Delta^{A}$ drops suddenly, where $P=0.4$, and $\Delta_{0}$ is the BCS zero-temperature energy gap. Then, $\Delta^{A}$ goes into the inhomogeneous $\mathrm{FF}$ state with $\mathbf{q} \neq 0$, decreases with the bias, and vanishes completely at $V=V_{c}$ where superconductivity is quenched, i.e. $\Delta_{\mathbf{q}}\left(V_{c}\right)=0$, as shown in Fig. 1(a). At $V=V^{*}$, there is a discontinuity for $\Delta^{A}$, implying that a firstorder phase transition from the homogeneous BCS phase to the FF phase exists in the system. The present result is quite different from that given in Ref. [9] where the inhomogeneous FF state was simply ignored. The chemical potential shift grows in the homogeneous BCS state with increasing the bias, and exbihits a jump at $V^{*}$, then increases slowly in the FF state till $V_{c}$, and is linear for $V \geqslant V_{c}$ in the normal state, as shown in Fig. 1(b). We have found that the free energy of the inhomogeneous $\mathrm{FF}$ phase is lower than the homogeneous BCS phase for $V_{c}>V \geqslant V^{*}$, suggesting that the FF state is stable. At $V=V^{*}$, the free energy of the FF state coincides with that of the homogeneous BCS state, revealing that the FF state can coexist with the BCS state at $V^{*}$.

The magnitude of momentum $\mathbf{q}$ of a Cooper pair is in general a function of bias voltage, as shown in Fig. 2. At $V<V^{*}$, the system is in the homogeneous BCS state, and thus $|\mathbf{q}|$ is zero; while for $V^{*} \leqslant V<V_{c},|\mathbf{q}|$ corresponding to the lowest free energy varies nonmonotonically with the bias, implying that the momenta of Cooper pairs in the stable, inhomogeneous SC state are not fixed. Note that there is a discontinuity for $|\mathbf{q}|$ at $V=V^{*}$, which is again the signature of the first-order phase transition.

Phase Diagram. - In the antiparallel configuration, a schematic phase diagram for the central superconductor in the $T-V$ plane could be obtained, as shown in Fig. 3 for $P=0.4$. There exist three phases: the homogeneous BCS phase with $\Delta$ constant $(\mathbf{q}=0)$ at low bias; the inhomogeneous FF phase with $\mathbf{q} \neq 0$ for $V^{*} \leqslant V<V_{c}$ at low temperature; the normal phase for $V \geqslant V_{c}$. Along the phase boundary from B to C, the homogeneous BCS phase coexists with the spatially modulated FF phase; along the $V_{c}$ boundary where the SC gap closes, there are two transitions: one is from the homogeneous BCS state to the normal state, and the other is from the spatially modulated FF state to the normal state, which are of
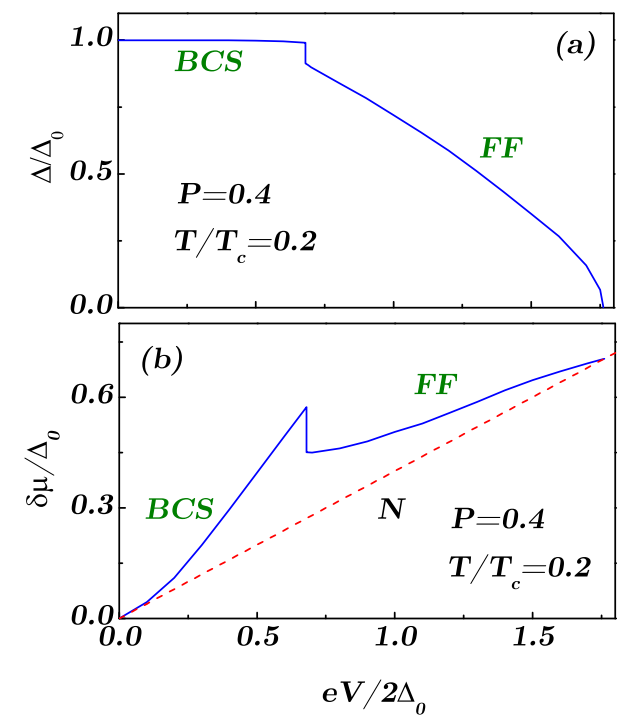

FIG. 1: (Color online) The bias dependence of the order parameter $\Delta^{A}$ (a) and the chemical potential shift (b) in the antiparallel alignment of magnetizations at $P=0.4, T / T_{c}=0.2$, where a discontinuity from the first-order phase transition is observed. The dashed line is for the normal state.

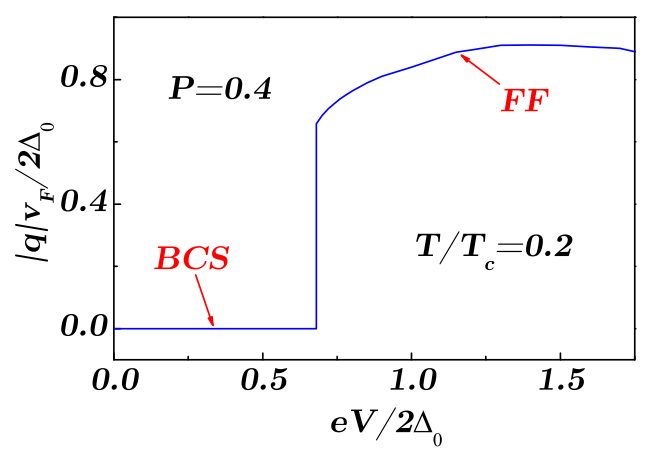

FIG. 2: (Color online) The bias dependence of the momentum of a Cooper pair in the antiparallel alignment of magnetizations at $P=0.4, T / T_{c}=0.2$, where a discontinuity is observed.

second-order. At point $\mathrm{C}$, three phases meet, implying it can be viewed as a Lifshitz point [27]; at point QCP where $V_{Q C P}$ satisfies $P e V_{Q C P} / 2 \Delta_{0}=0.754\left[=\delta \mu\left(V_{Q C P}\right)\right]$, a quantum phase transition (QPT) from the ordered $\mathrm{FF}$ state to the disordered normal state occurs at $T=0$.

$T M R$. - The total currents are given by $I^{F}=I_{0} N^{F}$, and $I^{A}=I_{0}\left(1-P^{2}\right) N^{A}$, where $I_{0}=2 \pi e|\widetilde{T}|^{2}\left(D_{j \uparrow}+D_{j \downarrow}\right)$, and $N^{F(A)}$ is given by Eq. (44). The differential conductance $G^{F(A)}$ and the tunneling magneoresistance $T M R$ are obtained by $G^{F(A)}=\frac{d I^{F(A)}}{d V}$ and $T M R=\frac{G^{F}}{G^{A}}-1$. The bias dependence of the tunnel conductance and the $T M R$ is shown in Fig. 4. The behavior of $G^{F}$ is consistent with that of Ref. [9], but $G^{A}$ and $T M R$ differ from those in Ref. [9] owing to the intervention of the FF state, where the oscillating behaviors for $G^{A}$ and $T M R$ are 


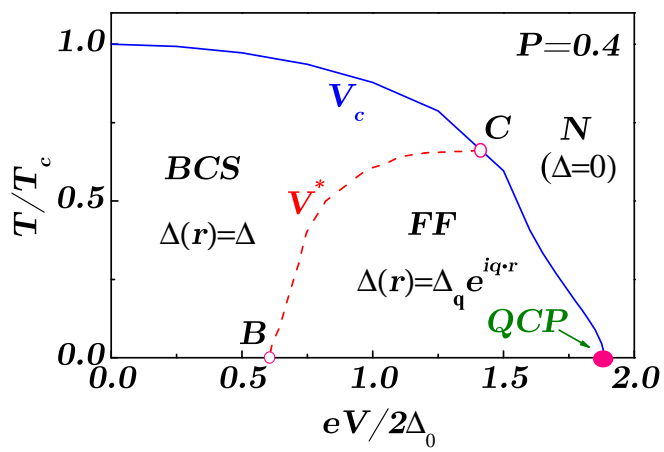

FIG. 3: (Color online) A schematic phase diagram of the central superconductor in the T-V plane for the antiparallel alignment of magnetizations at $P=0.4$. Three phases are observed: the homogeneous BCS state is separated by the $V^{*}$ boundary line via a first-order phase transition from the inhomogeneous FF state, while the $\mathrm{SC}$ states are separated by the $V_{c}$ boundary line via a second-order phase transition from the normal state. A quantum phase transition is sepcified at $V_{Q C P}$ (see context).
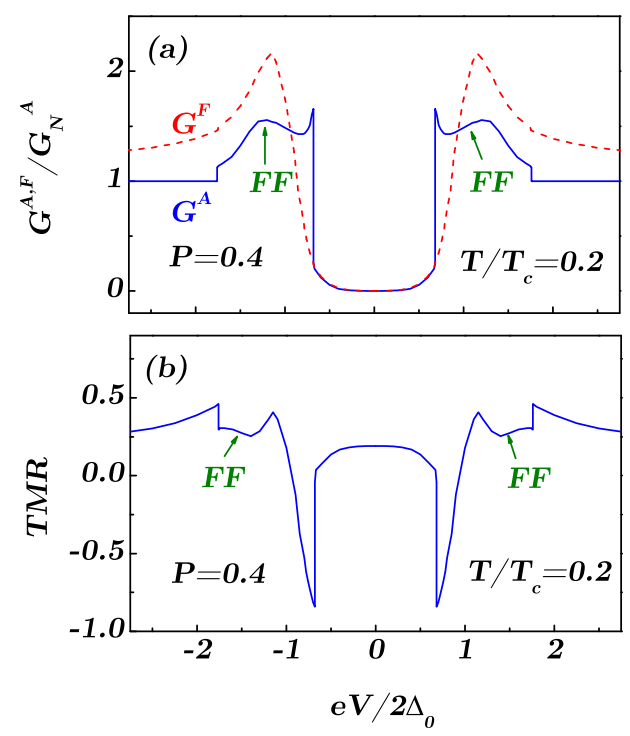

FIG. 4: (Color online) The bias dependence of the tunnel condanctance $G^{F, A}$ (a) and the magnetoresistance (b) at $P=0.4$, $T / T_{c}=0.2$, where $G_{N}^{A}$ is the conductance in the normal state for the antiparallel alignment. The oscillation characteristics are observed.

seen. These oscillation characteristics could be served as good tests for experimentally observing the inhomogeneous FF state, and are qualitatively consistent with the recent observation [20].

Quantum Criticality. - Since a phase transition from the inhomogeneous FF state to the normal state appears at $V_{Q C P}$, which just happens at $T=0$, it is nothing but a manifestation of the QPT 28]. At $V \lesssim V_{Q C P}$, the system is in an ordered ground state; while $V>V_{Q C P}$, it is in a disordered ground state, suggesting $V_{Q C P}$ is the quantum critical point, where the SC gap vanishes.
As $V$ approaches $V_{Q C P}$, the amplitude of SC order parameter, the chemical potential shift induced by the spin accumulation, as well as the difference of free energies between the SC and normal states vanish as $\Delta_{\mathbf{q}} / \Delta_{0}$, $\left[\delta \mu\left(V_{Q C P}\right)-\delta \mu(V)\right], F_{S}^{A}-F_{N}^{A} \sim\left|V-V_{Q C P}\right|^{z \nu}$ with the critical exponents $z \nu=1 / 2,1$ and 2. Obviously, such a QPT is of second-order. The present system offers a nice example of the QPT.

In summary, we have revisited the spin-dependent transport in $\mathrm{F} / \mathrm{S} / \mathrm{F}$ double tunnel junctions by taking the proximity effect into account. It is found that in the antiparallel configuration, the first- and second-order phase transitions, the inhomogeneous FF state, and quantum criticality can be revealed simultaneously in the central superconductor. The present study rectifies the previous result [9] where the FF state was simply ignored.

This work is supported in part by the NSFC (Grant Nos. 90103023, 10104015, 10247002).

[] * Corresponding author. E-mail: gsu@gscas.ac.cn.

[1] R. Meservey and P.M. Tedrow, Phys. Rep. 238, 173 (1994).

[2] M.A.M. Gijs and G.E.W. Bauer, Adv. Phys. 46, 285 (1997).

[3] G. Prinz, Science 282, 1660 (1998).

[4] J.S. Moodera et al., Annu. Rev. Mater. Sci. 29, 381 (1999).

[5] S.A. Wolf et al., Science 294, 1488 (2001).

[6] S. Das Sarma, American Scientist 89, 516 (2001).

[7] S. Maekawa and T. Shinjo (eds.), Spin-Dependent Transport in Magnetic Nanostructures (Taylor \& Francis, London and New York, 2002).

[8] M.J.M. de Jong et al., Phys. Rev. Lett. 74, 1657 (1995).

[9] S. Takahashi et al., Phys. Rev. Lett. 82, 3911 (1999).

[10] F. J. Jedema et al., Phys. Rev. B 60, 16549 (1999).

[11] Z. Zheng et al., Phys. Rev. B 62, 14326 (2000).

[12] Y. Tserkovnyak et al., Phye. Rev. B 65, 094517 (2002).

[13] N. Yoshida et al., Phys. Rev. B 63, 024502 (2000); N. Yoshida et al., Physica C 367, 185 (2002).

[14] B. Jin, G. Su, Q. R. Zheng, M. Suzuki, Phys. Rev. B 68, 144504 (2003).

[15] V.A. Vasko et al., Phys. Rev. Lett. 78, 1134 (1997); Z.W. Dong et al., Appl. Phys. Lett. 71, 1718 (1997); S.K. Upadhyay et al., Phys. Rev. Lett. 81, 3247 (1998); N.C. Yeh et al., Phys. Rev. B 60, 10522 (1999); A. Sawa et al., Physica C 339, 287 (2000).

[16] R.J. Soulen et al., Science 283, 85 (1998).

[17] T. Kontos et al., Phys. Rev. Lett. 86, 304 (2001).

[18] C.D. Chen et al., Phys. Rev. Lett. 88, 047004 (2002); J. Magn. Magn. Mat. 239, 141 (2002).

[19] J. Y. Gu et al., Phys. Rev. B 66, 140507(R) (2002).

[20] J. Johansson et al., J. Appl. Phys. 93, 8650 (2003).

[21] M. Johnson et al., Phys. Rev. Lett. 55, 1790 (1985); M. Johnson, ibid. 70, 2142 (1993); Science 260, 320 (1993); Appl. Phys. Lett. 65, 1460 (1994).

[22] P. Fulde and A. Ferrel, Phys. Rev. 135, A550 (1964).

[23] A. Larkin and Y. Ovchinnikov, Sov. Phys. JETP 20, 762 (1965). 
[24] R. Casalbuoni et al., Rev. Mod. Phys. 76, 263 (2004) and references therein.

[25] A.A. Abrikosov, Fundamentals of the Theory of Metals (North-Holland, Amsterdam, 1988), p.518.

[26] D. R. Heslinga et al., Phys. Rev. B 47, 5157 (1993).

[27] P. M. Chaikin, T. C. Lubensky, Principles of Con- densed Matter Physics (Cambridge University Press, Cambridge, 1995), p676.

[28] S. Sachdev, Quantum Phase Transitions (Cambridge University Press, Cambridge, 1999). 